\title{
ANTARES Status Report
}

\section{C.N. De Marzo*}

INFN and University, Bari, ITALY

On behalf of the ANTARES Collaboration - 'carlo.demarzo@ba. infn. iti'

\begin{abstract}
The aim of the ANTARES Project is the construction of an array of 900 optical modules to form a high-energy water Cherenkov neutrino telescope in the depth of Mediterranean Sea. The effective area is expected to be greater than $0.02 \mathrm{~km}^{2}$ for muons of energy greater than $10 \mathrm{TeV}$. It is optimized for detection of muons resulting from interactions of high-energy neutrinos of astrophysical origin. This detector will be built near Toulon (France) at $40 \mathrm{~km}$ off the coast, at a depth of $2400 \mathrm{~m}$. The detector will measure high energy neutrinos in a range from a few tens of $\mathrm{GeV}$ to the highest energies with good pointing resolution. Progress reports on construction and prototypes performance are given.
\end{abstract}

\section{Introduction}

Foundation of High Energy Neutrino Astronomy is one of the most promising challenge of Modern Astrophysics. Neutrinos are likely to be produced in several classes of astrophysical phenomena: from central engines of high-luminosity distant extra-galactic sources to galactic micro-quasars and supernova remnants. The high energy neutrino fluxes from these possible sources could be detected by a telescope having proper volume and effective area. Moreover diffuse fluxes produced, for instance, by unresolved neutrino sources should be detectable also [i], i2ind.

High-energy neutrinos can be produced by interactions of protons or photons on matter via the decay of pions. For example, in a supernova explosion there can be mechanisms of beam dumping led by proton accelerated in the magnetosphere or at the front of the shock wave. So the turbulent shells of supernova remnants can be a major galactic source of high-energy cosmic ray protons and, as a consequence, a source of neutrinos produced by proton interactions. In this kind of astrophysical sites high energy gamma rays are also produced by electromagnetic processes. Then the detection of high-energy neutrinos can be the only way for testing the hypothesis of hadronic production in sources. In favor of this possibility some observational hints have been published recently [푹]

\footnotetext{
${ }^{*}$ Speaker.
} 
Neutrinos are weakly interacting neutral particles that can escape from the depths of astrophysical objects and reach us while still pointing back to their sources. The Neutrino Astronomy is based on this property which promises to provide information on sites of the Universe not otherwise reachable by direct observation using photons or charged particles. The interaction cross sections of these probes are many orders of magnitude larger than those of the neutrinos, so they are impeded by absorption, energy degradation or magnetic deflection along the path implying that neutrino astronomy, once again, can be the only tool for studying those regions.

It is foreseen that various types of cosmic objects can be sources of high-energy neutrinos, starting with Active Galactic Nuclei (AGN) that are the most luminous objects in the Universe. To account for a luminosity evaluated as $10^{35} \div 10^{41} \mathrm{~W}$ a black hole of $10^{4} \div 10^{10}$ solar masses is hypothesized to power the energy production at the center of the AGN. Protons accelerated in the black hole accretion region of the AGN can produce high-energy neutrinos most likely. In addition, interacting with radiation in the jet of these

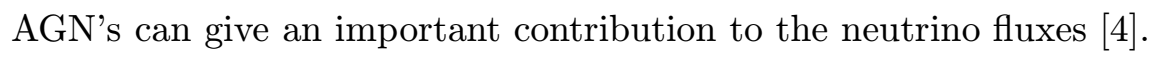

In the same way, binary systems containing a neutron star or a black hole are candidate as high energy neutrino sources. Besides electromagnetic processes, the intense luminosity of hard gamma-rays $\left(\mathrm{E}>10^{14} \mathrm{eV}\right)$ in these sources can be associated with high-energy neutrinos produced through inelastic hadronic interactions.

Other possible sources of high energy neutrinos to look for are Gamma Ray Bursts (GRB), whose nature has still many aspects to be clarified.

To establish High Energy Neutrino Astronomy suitable telescopes are needed. Currently Cherenkov light detection in large volumes of water deep under sea, lake or ice, appears the only viable possibility. Upward-going muon-neutrinos produced in interactions with surrounding matter are selected in order to cope with the background given by the downward-going cosmic ray muons.

Some electronic detectors of the past generation - for instance, Frejus, Macro and others - have put upper limits to neutrino fluxes, both from diffuse and point-like sources

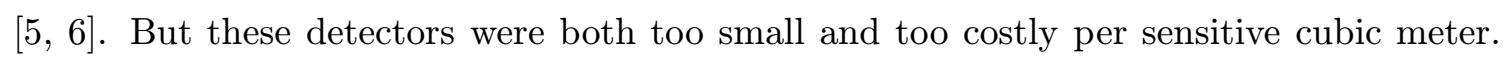
Given that volume detector required to start neutrino astronomy is of order $10^{8} \div 10^{9}$ cubic meters, the Cherenkov light detection is the only possible technique because of its low cost per cubic meter of detector and because it does not need huge underground laboratories. But, of course, this is a new and difficult technique that needs to be further developed to its full potentiality, even though an international community has already devoted great efforts to build the first generation of under-water neutrino telescopes. Notably this has

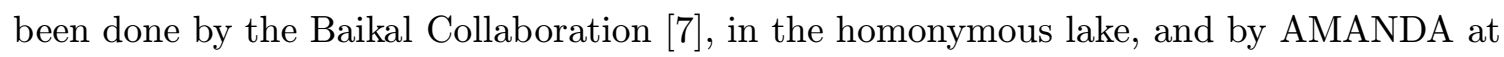
the South Pole [i్⿴囗十).

The ANTARES experiment follows on this line. It is at present under construction with the aim, primarily, at studies in the above fields by the detection of high-energy neutrinos with good pointing resolution. This can provide information on the mechanisms of high-energy neutrino sources even at cosmological distances [i

Besides high energy astrophysical neutrino sources, the search for WIMP's is another item of the ANTARES scientific program. The best candidates are neutralinos that can 


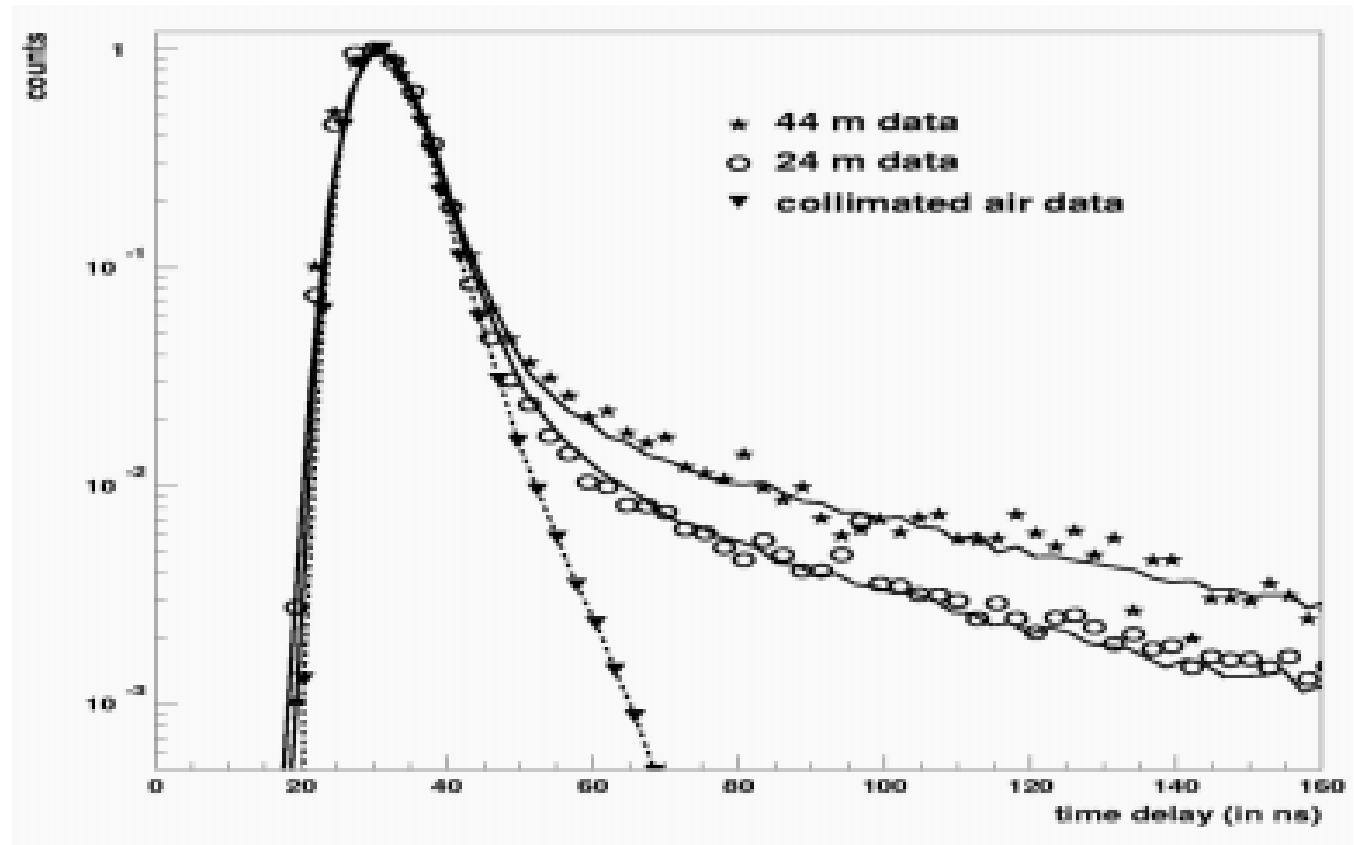

Figure 1: Distribution of photon arrival times for distances $24 \mathrm{~m}$ and $44 \mathrm{~m}$ between a photomultiplier and a source. The data for $44 \mathrm{~m}$ are normalized to the results for $24 \mathrm{~m}$. Arrival time distributions for two distances and calibration in air are superimposed.

be gravitationally trapped inside celestial bodies. Hence they could accumulate inside the Earth, Sun or in the galactic center. After annihilation they would produce neutrinos with energy below $1 \mathrm{TeV}$ allowing detection by the telescope.

And last we cannot exclude a chance of serendipitous discoveries that is always possible when new intervals of physical variables become open to observation.

\section{The ANTARES site}

The site of the ANTARES telescope is about $40 \mathrm{~km}$ from the coast near Toulon (France) at $2400 \mathrm{~m}$ depth. This site has been characterized by an extensive explorative programme that has measured environmental parameters and their dependence with time. Figures $\underline{1}_{1}^{1}$ to $\bar{\beta}_{1}^{i}$ show some optical properties of the sea water at the ANTARES site, i.e. light transmission properties, bio-luminescence and bio-fouling. The distance travelled by photons, and hence detector efficiency, strongly depends on water transparency. Light scattering in sea-water affects the photon arrival time to PMT's, hence the angular resolution of the detector. Light pulses from a light emitting diode (LED) were measured at distances of 44 and $24 \mathrm{~m}$ by a 10" fast photo-multiplier (PMT). The LED luminosity was adjusted such as to give a constant PMT anode current. The distribution of photon arrival times (Fig.1) shows a peak from direct photons with a tail due to light scattering effects. It was found that for blue light $(\lambda \sim 470 \mathrm{~nm})$ the absorption length is around $55 \mathrm{~m}$, the scattering length of 

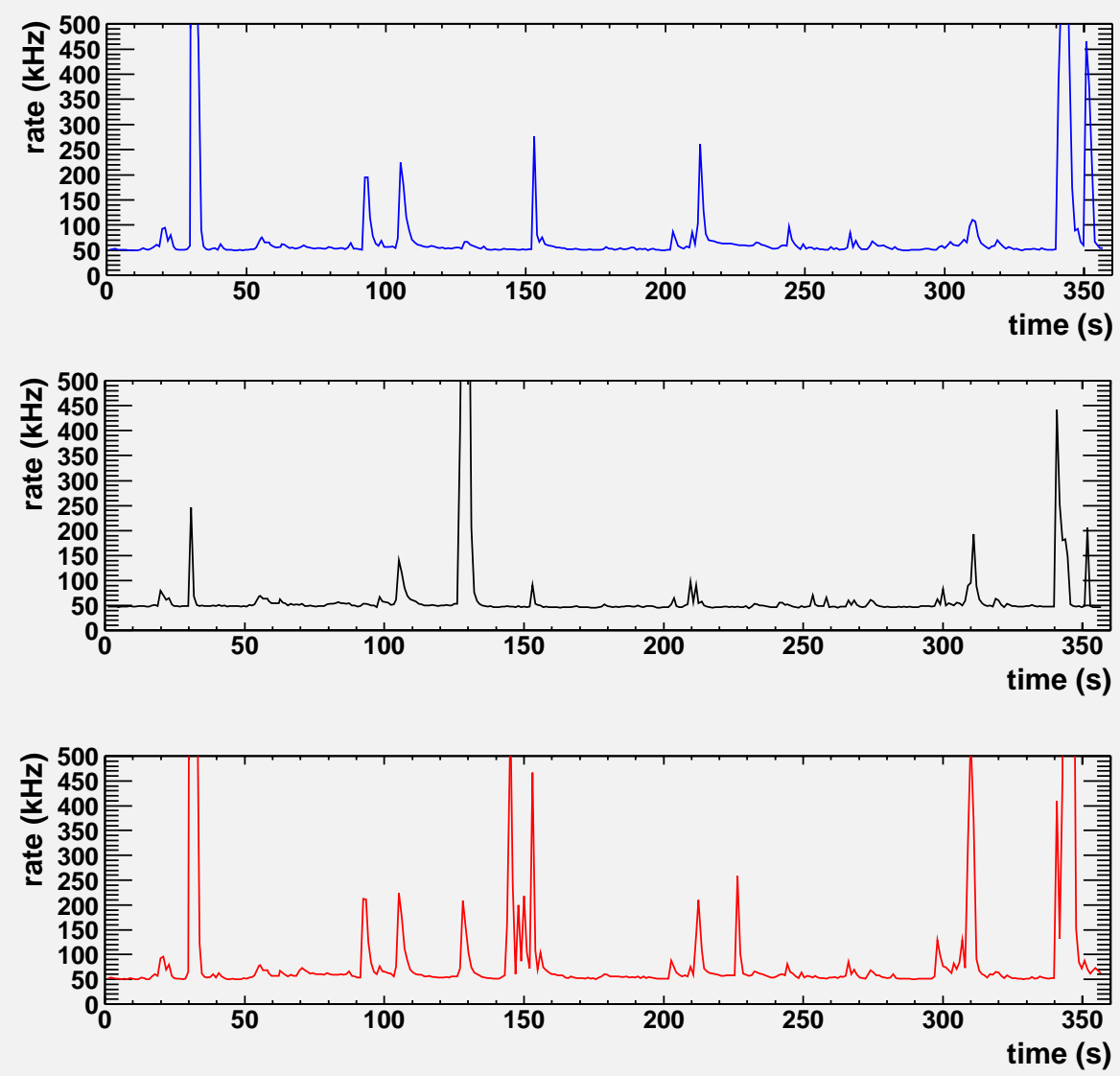

Figure 2: Variations with time (seconds) of the optical background counting rate $(\mathrm{kHz})$.

order $200 \mathrm{~m}$ and the mean value $\cos \theta$ of about 0.05 - where $\theta$ is the photon scattering angle - while the absorption length for UV light $(\lambda \sim 370 \mathrm{~nm})$ is around $25 \mathrm{~m}$.

The rates of background signals and their correlations have been measured. Figure $\overline{2}$ shows a typical result: a background of $60 \mathrm{kHz}$ on a 10" PMT, mainly due to radioactive $40 \mathrm{~K}$, varies slowly over a time scale of hours up to $250 \mathrm{kHz}$. Bursts of a few seconds due to bio-luminescence appear superimposed on this baseline with rates up to several MHz.

Sediment as well as a layer of micro-organisms (bio-fouling) can settle on optically active surfaces. Both processes can decrease the light collection efficiency of PMTs placed inside the pressure resistant glass sphere constituting the Optical Modules (OM's). In order to investigate this phenomenon at the ANTARES site, five PIN diodes illuminated by a blue LED were fixed at angles from $50^{\circ}$ to $90^{\circ}$ from the vertical axis. Results over 240 monitoring days are shown in figure ㅆ.1. For the horizontally positioned diode - viewed through a vertical OM surface - the loss of light intensity due to fouling (a few percent after 1 year, then saturates) is small compared to the upward viewing parts of the OM surface. 


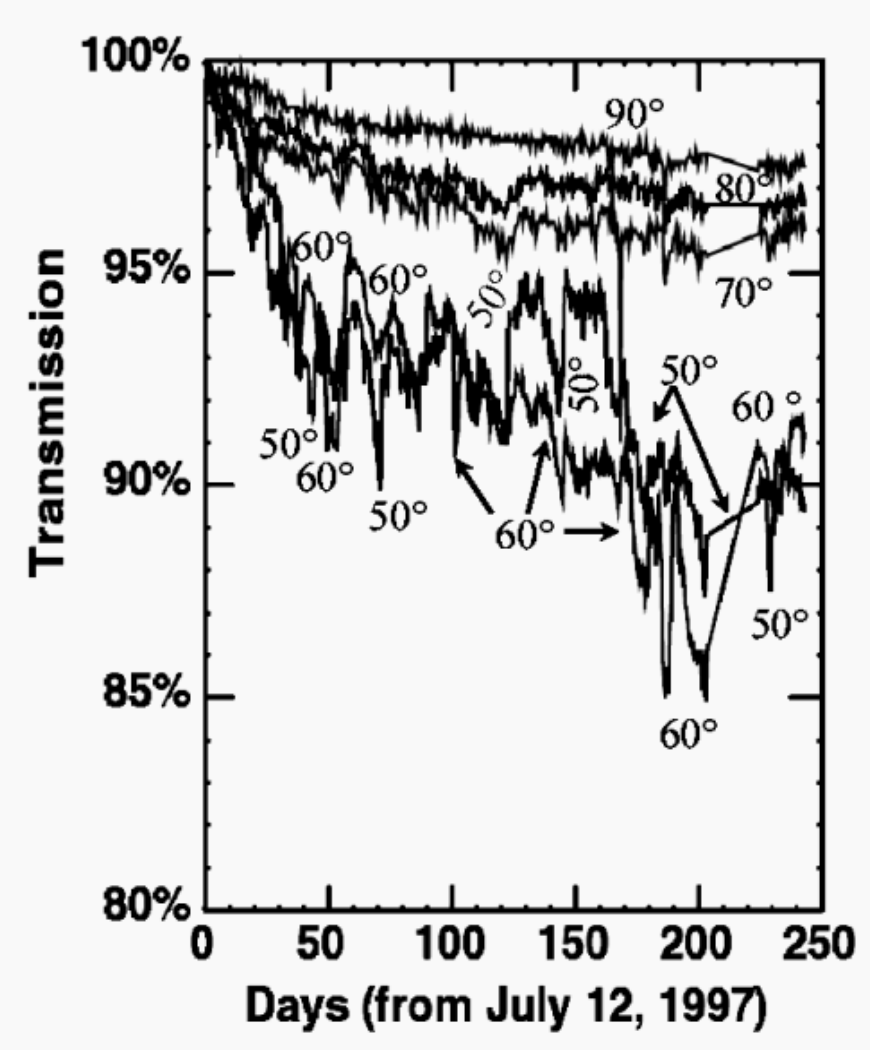

Figure 3: Decreasing of OM transparency with time (relative to the first day) relative to the first day at various angles over 8 months.

After these and some more other measurements the site has been thoroughly characterized. It was found that the optical properties of the sea-water are adequate, the temperature is stable on $13^{\circ} \mathrm{C}$ at the depth of $2400 \mathrm{~m}$, and the currents are slow $(\sim 5$ $\mathrm{cm} / \mathrm{s})$.

\section{The ANTARES telescope structure}

The ANTARES telescope will consist of an array of 12 vertical strings (see Fig.'ín). Each string consists of an electro-optical-mechanical cable of about $460 \mathrm{~m}$ length, anchored on the seabed by means of a ballasted bottom string socket (BSS) and kept vertical by means of a buoy at the upper end. The cable supports $75 \mathrm{OMs}$, arranged in 25 triplets or storeys spaced $14.5 \mathrm{~m}$ apart, with the lowest $100 \mathrm{~m}$ of cable without instruments. Each storey has an electronic Local Control Module (LCM). The separation between adjacent strings is approximately $70 \mathrm{~m}$.

An OM consists of a 17" watertight glass sphere containing a 10" Hamamatsu PMT and associated electronics. The PMT is housed in a mu-metal wire cage to shield it from the Earth's magnetic field. The three OMs have their PMT face outward from the cable and oriented downwards at $45^{\circ}$ respect to the vertical. The 25 storeys on each string are 


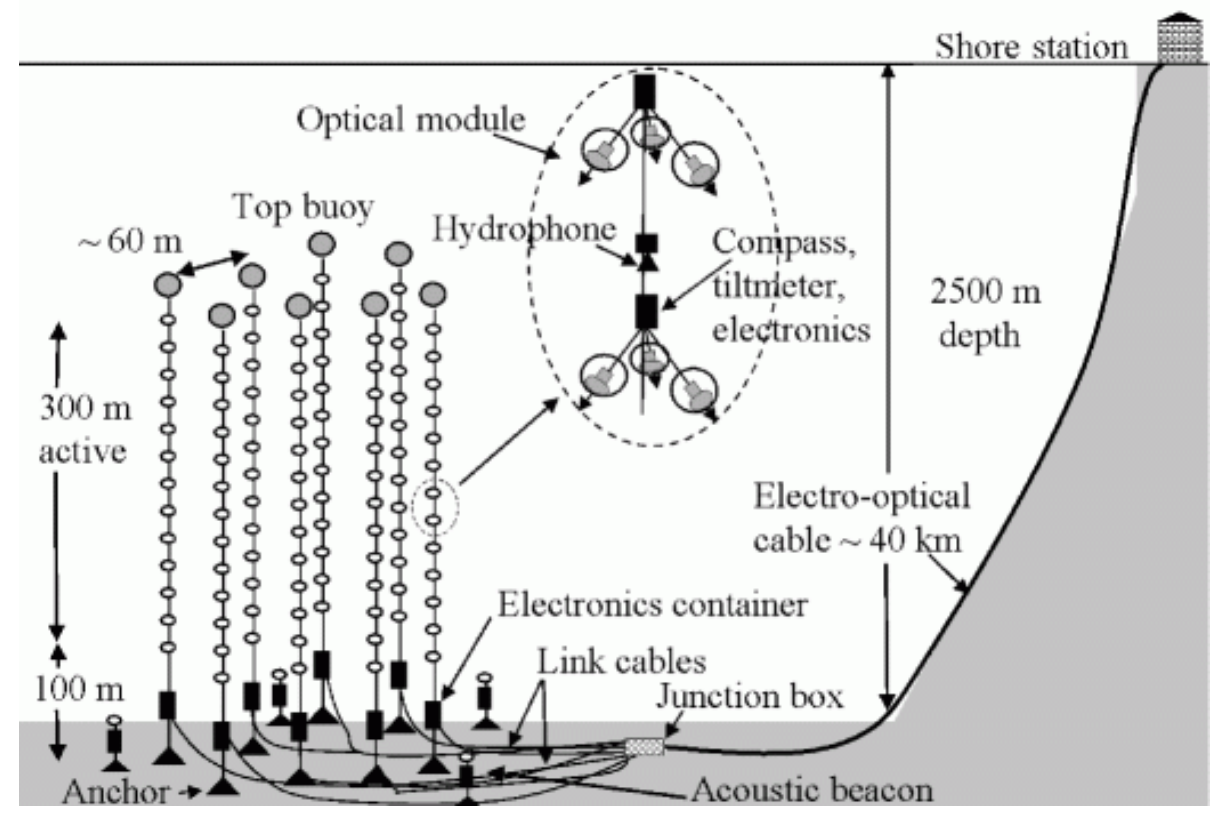

Figure 4: Schematic view of the ANTARES apparatus with strings and main subsystems.

divided into 5 sectors of 5 storeys each. The PMTs look downward to avoid sedimentation. This also reduces background from the direct flux of muons produced by cosmic rays in the atmosphere above the detector.

The detector is connected to shore by an electro-optical (E/O) cable. This ends at an interface, the Junction Box (JB), which distributes power and control signals to - and collects data from - the individual strings. Each string has its own short E/O cable to the $\mathrm{JB}$, which is connected to the JB after deployment by means of a submarine device.

The read-out system provides the digitization of the analogue PMT signals. The dataacquisition system controls the transfer of the data through a high-bandwidth network to an on-shore PC farm for on-line filtering before off-line track reconstruction and analysis.

Extensive Monte Carlo simulations have been used to estimate the expected performance of the detector telescope.

\section{The ANTARES telescope performance}

Pointing resolution in neutrino telescopes is important for searching point-like sources. In ANTARES the pointing resolution for muon tracks is determined by the timing resolution of the detector, which is better than $1 \mathrm{~ns}$. At energies above $10 \mathrm{TeV}$ the muon direction is close to the parent neutrino direction, and the accuracy of the neutrino direction is expected to be better than $0.3^{\circ}$ including light scattering effects. At muon energies below $10 \mathrm{TeV}$ the angular error is dominated by spread in the neutrino-muon relative angle (see Fig. 登).

The measurement of the muon energy can be deduced from the charge of PMT signals. This parameter is important particularly for neutrino diffuse flux search. In ANTARES 


\section{Angular resolution}

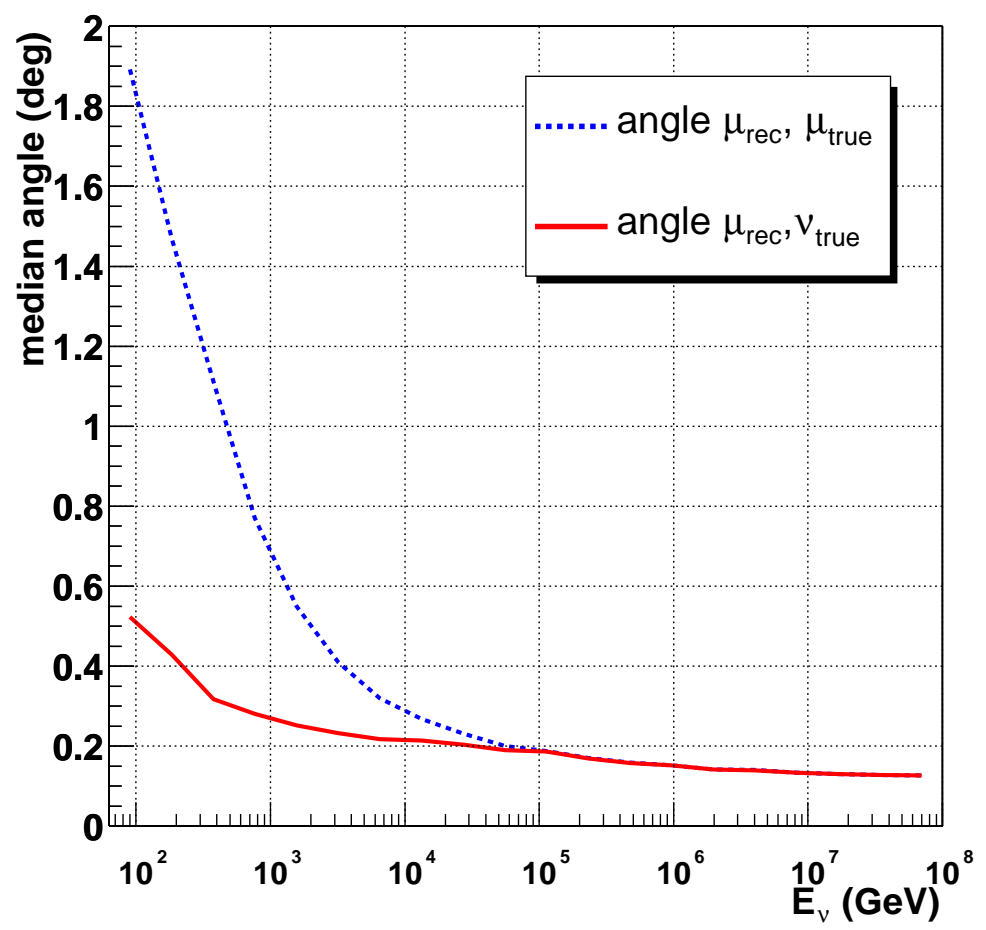

Figure 5: Angular resolution of the reconstructed muon and of its the parent neutrino as a function of the neutrino energy.

it can be measured with a precision better than a factor 3 for muons in the range above $10 \mathrm{TeV}$. For contained events below $100 \mathrm{GeV}$ the muon energy can be evaluated more accurately from the measured muon range.

A significant advantage of the ANTARES telescope - located at a latitude of $42^{\circ}$ - is the large solid angle covered: $3.5 \pi$ sr, taking the rotation of the Earth into account. The galactic center will be visible most of the time. The simultaneous overlap in solid angle with AMANDA is $0.5 \pi$ sr.

\section{The ANTARES status of construction}

The ANTARES Collaboration is actively constructing the neutrino telescope. The electrooptical cable to shore and the Junction Box have been deployed and connected. Recently a prototype Sector Line have been deployed and recovered successfully, demonstrating the feasibility of these operations. Working on this prototype other important functions have been demonstrated: power distribution works and is stable; slow control works; data from OM's and monitoring devices are read; single rates from different OM's are consistent [i] $\left.1 \overline{0}_{0}^{0}\right]$.

During these tests a wealth of data have been transmitted to shore, mainly on environmental parameters, which are still under investigation.

Deployment of the 12 strings will start at the beginning of 2005 and will be completed in about 2 years. 


\section{Conclusion}

The aim of the ANTARES Collaboration is to construct a large water Cherenkov detector in the deep Mediterranean Sea, optimized for detection of muons resulting from interactions of high-energy neutrinos of astrophysical origin.

The detector will also allow us to search for neutralino dark matter.

In the selected site adequate environmental parameters have been measured.

Prototype strings have been deployed and recovered successfully. Tests on the prototypes demonstrate that the construction of this detector is well feasible.

Full detector deployment will be completed before the end of 2006 .

\section{References}

[1] T.K. Gaisser, F. Halzen, T. Stanev, Phys. Rep. 258(1995)173

[2] J.G. Learned and K. Mannheim, Ann. Rev. Nucl. Sci. 50(2000)679

[3] R. Enomoto et al. Nature 416(2002)823-26

[4] K. Mannheim and P.L. Biermann, Astron. Astrophys. 253(1992)L21

[5] W. Rhode et al. Astropart. Phys. 4(1996)217-225

[6] M. Ambrosio et al. Ap. J. 546(2001)1038

[7] http://www.ifh.de/baikal/

[8] http://amanda.berkeley.edu/

[9] The ANTARES Collaboration, "A deep-sea telescope for high energy neutrinos", as-tro-ph/9907432; see also http://antares.in2p3.fr/

[10] V. Bertin, "Status of the ANTARES Neutrino Telescope", TAUP Conference, Seattle,USA, September 2003 